\title{
Individualized diagnosis of major depressive disorder via multivariate pattern analysis of thalamic sMRI features
}

Hanxiaoran $\mathrm{Li}^{1,2,3}$, Sutao Song ${ }^{4^{*+}}$, Donglin Wang ${ }^{1,2,3,5^{*+}}$, Zhonglin Tan ${ }^{6}$, Zhenzhen Lian ${ }^{1,2,3}$, Yan Wang ${ }^{1,2,3,5}$, Xin Zhou ${ }^{1,2,3}$ and Chenyuan Pan ${ }^{1,2,3}$

\begin{abstract}
Background: Magnetic resonance imaging (MRI) studies have found thalamic abnormalities in major depressive disorder (MDD). Although there are significant differences in the structure and function of the thalamus between MDD patients and healthy controls (HCs) at the group level, it is not clear whether the structural and functional features of the thalamus are suitable for use as diagnostic prediction aids at the individual level. Here, we were to test the predictive value of gray matter density (GMD), gray matter volume (GMV), amplitude of low-frequency fluctuations (ALFF), and fractional amplitude of low-frequency fluctuations (fALFF) in the thalamus using multivariate pattern analysis (MVPA).

Methods: Seventy-four MDD patients and 44 HC subjects were recruited. The Gaussian process classifier (GPC) was trained to separate MDD patients from HCs, Gaussian process regression (GPR) was trained to predict depression scores, and Multiple Kernel Learning (MKL) was applied to explore the contribution of each subregion of the thalamus.

Results: The primary findings were as follows: [1] The balanced accuracy of the GPC trained with thalamic GMD was $96.59 \%(P<0.001)$. The accuracy of the GPC trained with thalamic GMV was $93.18 \%(P<0.001)$. The correlation between Hamilton Depression Scale (HAMD) score targets and predictions in the GPR trained with GMD was 0.90 $\left(P<0.001, r^{2}=0.82\right)$, and in the GPR trained with GMV, the correlation between HAMD score targets and predictions was $0.89\left(P<0.001, r^{2}=0.79\right)$. [2] The models trained with ALFF and fALFF in the thalamus failed to discriminate MDD patients from HC participants. [3] The MKL model showed that the left lateral prefrontal thalamus, the right caudal temporal thalamus, and the right sensory thalamus contribute more to the diagnostic classification.

Conclusions: The results suggested that GMD and GMV, but not functional indicators of the thalamus, have good potential for the individualized diagnosis of MDD. Furthermore, the thalamus shows the heterogeneity in the structural features of thalamic subregions for predicting MDD. To our knowledge, this is the first study to focus on the thalamus for the prediction of MDD using machine learning methods at the individual level.
\end{abstract}

\footnotetext{
*Correspondence: sutao.song@sdnu.edu.cn; wangdl@hznu.edu.cn

†'Sutao Song and Donglin Wang contributed equally to this work.

${ }^{4}$ School of Information Science and Engineering, Shandong Normal

University, 1\#, University Rd, Changqing District, Jinan 250358, China

'Institutes of Psychological Sciences, College of Education, Hangzhou Normal

University, 2318\#, Yuhangtang Rd, Hangzhou 311121, China

Full list of author information is available at the end of the article
}

(C) The Author(s). 2021 Open Access This article is licensed under a Creative Commons Attribution 4.0 International License, which permits use, sharing, adaptation, distribution and reproduction in any medium or format, as long as you give appropriate credit to the original author(s) and the source, provide a link to the Creative Commons licence, and indicate if changes were made. The images or other third party material in this article are included in the article's Creative Commons licence, unless indicated otherwise in a credit line to the material. If material is not included in the article's Creative Commons licence and your intended use is not permitted by statutory regulation or exceeds the permitted use, you will need to obtain permission directly from the copyright holder. To view a copy of this licence, visit http://creativecommons.org/licenses/by/4.0/ The Creative Commons Public Domain Dedication waiver (http://creativecommons.org/publicdomain/zero/1.0/) applies to the data made available in this article, unless otherwise stated in a credit line to the data. 
Keywords: Major depressive disorder (MDD), Thalamus, Multivariate pattern analysis (MVPA), Individualized diagnosis

\section{Background}

Major depressive disorder (MDD) is a common disorder that is associated with a series of clinical symptoms, such as depressed mood, loss of energy, difficulty with concentration and short-term memory and decision making, etc. [1]. As a mental disorder, MDD may create an enormous burden and harm for patients and society. Current clinical diagnostic approaches for MDD are based mainly on the subjective assessment of symptoms through clinician interviews with patients. Psychiatric diagnosis depends largely on statements by the patients and their relatives, psychometrists' use of many rating scales, and psychiatrists' personal experiences. These diagnostic methods, however, do not involve any biological or physiological markers and therefore are not objective enough, which may lead to misdiagnosis [2]. To avoid misdiagnosis and achieve better treatment outcomes, objective and individualized diagnostic approaches are urgently needed.

In searching for biomarkers useful for objective diagnosis of MDD, many studies have contributed a lot to the identification of biological correlates of MDD patients in recent years [3-5]. From a special perspective, our recent study demonstrated abnormalities in thalamus in MDD patients [6]. Also, results from many other studies suggest that thalamic abnormalities might be important potential biomarkers of MDD [7-9]. The clinical symptoms of MDD may arise, at least in part, through the corresponding dysfunctions of thalamus and thalamus-related neural circuits [10].The thalamus is not only a sensory relay station involved in emotion, memory, and arousal [11], but also plays a central role in the ongoing cortical function [12], and is a key central region, which can integrate all kinds of information being processed by the whole cerebral cortex [13]. Meanwhile, the thalamus is a part of the salience network, which has been proved to have a central role in MDD [14]. Both structural and functional abnormalities of thalamus were found in patients with MDD. Patients with MDD were shown to have reduced fractional anisotropy values in the prefrontal lobe portion of the left anterior thalamic radiation and increased thalamic blood flow velocity compared with healthy people [15-17]. Other studies have demonstrated decreased left thalamic volume, a contracted shape on ventral aspects of the left thalamus and decreased gray matter volume (GMV) in the right thalamus $[16,18,19]$ or in the bilateral thalamus [20], and increased gray matter density (GMD) in the thalamus [21] in MDD patients, while some studies have shown larger thalamic volume, which was seen only in first-episode medication-naive patients. The results of these studies on thalamic GMD and GMV are inconsistent, and these results may be affected by age, severity of depression, and treatment. Thalamic structural abnormalities have been found in MDD patients of different ages. In adolescents with MDD, GMV in the thalamus is inversely related to the severity of self-reported symptoms and decreases with age, while healthy adolescents show increases with age [22]. In elderly patients with depression, the volume of the thalamus is smaller than that in normal people $[17,23]$. In terms of the severity of depression, although there is no significant correlation between depression scores and brain structure volume, higher depression scores have indicated more thalamic shape abnormalities [17],while some studies demonstrated that the severity of mild depressive symptoms was associated with reduced gray matter volume in the thalamus [24]. Moreover, thalamic abnormalities have been found in people at high risk of depression. In people with subthreshold depression, the GMV in the thalamus was increased [25]. In a study of healthy people with cognitive vulnerability to depression, it was found that these subjects had a smaller right thalamus than MDD patients [19]. In addition, the thalamus may be related to antidepressant therapy. A multiple regression analysis revealed that pretreatment smaller GMV in the left thalamus was associated with a poorer response to electroconvulsive therapy (ECT) and lower fractional amplitude of low-frequency fluctuations (fALFF) in the left thalamus [26]. Moreover, some studies have demonstrated that the thalamus may play an important role in MDD via thalamocortical circuits. A recent review of previous studies reported that thalamocortical circuits are candidates for controlling the activity of the default network, including task-suppression effects [27]. Thalamocortical circuits are anatomically well situated to exert a broad influence within and between cortical networks and to act as modulatory hubs [28]. Additionally, dysregulation of thalamocortical circuits might increase the risk of certain forms of mental illness, including MDD [27]. Using group statistical analysis methods, the abovementioned studies have provided strong evidence that thalamic abnormalities are closely relevant to MDD. Still, it remains unknown whether the thalamus could be used as proper feature to identify MDD patients at individual-level.

To predict individual cases, multivariate pattern analysis (MVPA) techniques could differentiate MDD 
patients from healthy controls (HCs) using magnetic resonance imaging (MRI) at the individual level. MVPA has been proven to be more sensitive and more informative about the organization of the cortex than univariate analysis with the general linear model (GLM). MVPA provides an investigation of different brain states that may be produced by a cortical field or systems, thus increasing the amount of information decoded from brain activities [29]. In recent years, a considerable number of studies have built support vector machine (SVM) models to predict the diagnosis of MDD or bipolar disorder (BD), MDD onset, refractory MDD patients, and treatment response to different types of antidepressant therapy, including electroconvulsive therapy, medication therapy and cognitive behavioral therapy, with over $70 \%$ accuracy by using structural magnetic resonance imaging (sMRI) or resting-state functional magnetic resonance imaging (rs-fMRI) information [30-38]. In addition, Gaussian process classification (GPC) has also been used to recognize MDD, BD, and remitted MDD patients using fMRI (e.g., amplitude of low-frequency fluctuations (ALFF) and fALFF) or sMRI (e.g., GMD) features with over $69 \%$ accuracy [7,39-45]. GPC is a supervised machine learning approach similar to SVM that provides the added benefit of predictive probabilities of class membership [46]. These results illustrate that MVPA methods show outstanding performance in individually discriminating MDD patients from healthy people and patients with other mental disorders.

Although previous MRI studies have demonstrated thalamic abnormalities in MDD and MVPA methods have shown good performance in individually recognizing MDD patients, to date, there has been no research on the individualized diagnosis of MDD using imaging features of the thalamus. Hence the present study is intended to focus on the thalamus and employ MVPA to predict MDD at the individual level. We were to use two MVPA methods, i.e., GPC and Gaussian process regression (GPR), to examine the potential predictive capacity of structural and rs-fMRI features of the thalamus. Besides, to explore which subregions of the thalamus contribute more to the diagnostic classification of MDD, a sparse version of Multiple Kernel Learning (MKL) was to be applied to explore the contribution of each subregion [47]. We hypothesized that the MRI features of the thalamus would be biomarkers for individualized diagnosis of MDD. More specifically, the predictive potential of both GPCs and GPRs trained with two structural features, i.e., GMD and GMV, and two rs-fMRI features, i.e., ALFF and fALFF, of the thalamus would be expected to bring interesting results for this hypothesis. We also hypothesized that the thalamic subregions would contribute differently to the individualized diagnostic classification of MDD.

\section{Methods \\ Participants}

In this study, 118 subjects were recruited, including 74 MDD patients (MDD group) and 44 healthy volunteers as a control group (HC group). Previous studies have shown that education level is a strong predictor of MDD and therefore should be strictly controlled for in the data analysis [48-52]. Because it was difficult to match, education level was included as a covariate and controlled for with statistical techniques during data processing in the present study.

MDD patients (49 female and 25 male patients with an average age of $26.53 \pm 8.56$ years) were recruited from the Department of Psychiatry of the Seventh People's Hospital of Hangzhou and the Department of Psychiatry of the Second People's Hospital of Hangzhou. All enrolled patients met the following criteria: [1] met the International Classification of Diseases, 10th Revision (ICD-10) criteria for MDD [2]; had no history of medication or physiotherapy for at least 1 month before recruitment or were taking only selective serotonin reuptake inhibitor (SSRI) antidepressants $\leq 1$ week [3]; had a Hamilton Depression Scale (version: 24 Items; HAMD-24) total score $\geq 20$; and [4] were $18-65$ years of age. There was no restriction on sex.

Healthy subjects $(28$ female and 16 male subjects with an average age of $29.34 \pm 12.42$ years) were recruited from universities in Hangzhou and communities near the hospitals by posters and internet announcements. The inclusion criteria were as follows: [1] did not meet the ICD-10 "depression episode" diagnostic criteria, had no family history of mental illness, and had not taken any medications at least 1 month before recruitment [2]; had a HAMD-24 total score $\leq 8$; and [3] were aged $18-65$ years.

Both MDD and HC subjects were right-handed Han Chinese individuals. Participants were excluded if they met any of the following criteria: a history of or current organic brain diseases, abuse of or dependence on psychoactive substances, schizophrenia or other psychiatric disorders, depressive episodes with psychotic symptoms or suicidal behavior, serious physical diseases, or any contraindications for MRI, and for women, pregnancy or lactation.

This study was approved by the ethics committee of the Institutes of Psychological Sciences, Hangzhou Normal University. All methods were performed in accordance with the relevant guidelines and regulations. All patients' legally authorized representatives and the controls provided written informed consent before participating in the study procedures.

\section{MRI data acquisition}

Three-dimensional MR imaging was acquired using a GE $3 \mathrm{~T}$ scanner (MR750, GE Medical Systems, 
Milwaukee, WI) with a 32-channel radio frequency coil at the Center for Cognition and Brain Disorders (CCBD), Hangzhou Normal University (HZNU). Foam filling was used to reduce head movement for all subjects. During scanning, the subjects were asked to relax and remain still. Using a magnetization-prepared rapid acquisition gradient-echo sequence, three-dimensional T1-weighted anatomical images were obtained in the sagittal orientation $(\mathrm{TR}=9 \mathrm{~ms}, \quad \mathrm{TE}=3.664 \mathrm{~ms}, \quad \mathrm{FOV}=$ $240 \times 240 \mathrm{~mm}^{2}, \quad$ matrix $=300 \times 300, \quad$ flip angle $=13^{\circ}$, thickness $=0.8 \mathrm{~mm}$, acquisition time $=13 \mathrm{~min} 37 \mathrm{~s}$ ). $\mathrm{fMR}$ images were acquired using a gradient-recalled echoplanar imaging sequence $(\mathrm{TR}=2000 \mathrm{~ms}, \mathrm{TE}=22 \mathrm{~ms}$, FOV $=240 \times 240 \mathrm{~mm}^{2}$, matrix $=96 \times 96$, flip angle $=77^{\circ}$, slice thickness $=2.5 \mathrm{~mm}$, no interslice gap, and 240 volumes).

\section{Data processing}

\section{MRI data preprocessing}

All datasets were preprocessed via DPABI_V3.1 (a toolbox for Data Processing \& Analysis for Brain Imaging) [53].

Structural data were segmented into GMV, GMD, white matter volume, white matter density, cerebral spinal fluid volume, and cerebral spinal fluid density. "Dartel+segment" was applied for normalization to the Montreal Neurologic Institute (MNI) space. Images were smoothed with an 8-mm full-width at half-maximum (FWHM) Gaussian kernel.

The following procedures were included in the rsfMRI data preprocessing: [1] removal of first 10 volumes [2]; slice timing correction [3]; head motion correction [4]; coregistration of T1 images to the averaged EPI image [5]; spatial normalization to standard Montreal Neurological Institute (MNI) space using "Dartel+segment" [6]; regression of head motion effects with the Friston-24 parameter model (all the subject's head motions were lower than our criteria of $2 \mathrm{~mm}$ and $2^{\circ}$ ) and regression of head motion, white matter (WM) and cerebrospinal fluid (CSF); and [7] removal of linear trends.

\section{Features used for classification and prediction}

DPABI was used to make the whole-thalamus mask [53] and calculate the GMV, GMD, ALFF, and fALFF values. The GMV, GMD, ALFF, and fALFF values in the thalamus were extracted as regression and classification features.

GMV and GMD are the important indicators of brain structure changes. Many MRI studies found that the abnormal brain structure changes in MDD [54, 55]. These two indicators were obtained through segmenting the structural images using "Dartel+segment".

ALFF and fALFF reflect the neural activity of the brain. The abnormal levels of ALFF and fALFF may be related to MDD [56-59]. ALFF/fALFF, which are important indicators, are used to detect the local intensity of spontaneous fluctuation of the blood-oxygen-leveldependent (BOLD) signal [60], and the change in local intensity of the BOLD signal depends on the spontaneous fluctuation of regional cerebral blood flow. Thus, increases in ALFF/fALFF may indicate excessive neurological activity in the brain, while decreases in ALFF/ fALFF may indicate insufficient neurological activity [61-63]. A ratio of the low-frequency amplitude within $0.01-0.1 \mathrm{~Hz}$ was computed at each voxel to obtain the ALFF and fALFF. The maps were smoothed by $8-\mathrm{mm}$ FWHM Gaussian kernel.

\section{Pattern analysis}

In this study, GPC was built for pattern classification, and GPR was built for HAMD score prediction using the Pattern Recognition for Neuroimaging data Toolbox (PRoNTo) toolbox (http://www.mlnl.cs.ucl.ac.uk/pronto) [64]. GPR has been widely used in supervised machine learning due to its flexibility and inherent ability to describe uncertainty in function estimation [65]. A mask of the thalamus was firstly added to limit the brain region for analysis, and the BrainnetomeAtlas which divided the thalamus into 16 subregions was added as a secondary mask (see Fig. 1) [66]. For every subregion, the signal in each voxel was extracted and concatenated as a feature vector. A vector was associated to a label (i.e. MDD or $\mathrm{HC}$ ). Then, a linear kernel was built from the feature vectors for each region. The computed kernels were added to obtain a whole thalamus linear kernel. The kernel and its associated labels were used to train the model and estimate the model parameters. The model can then give an associated predicted label for a new data [47]. No parameters need to be optimized during the model training. Fivefold cross-validation was used to evaluate the generalization performance of the models. Because of the imbalance between the number of MDD patients and $\mathrm{HC}$ subjects, balanced accuracy $\left(a c c^{b a l}=\frac{1}{C} \sum a c c_{c}\right.$, subscript "c" would be the number of the class) was used to evaluate the performance of each classifier. A 1000permutation test was performed to determine statistical significance, and cross-validation was repeated for each permutation.

Besides, the MKL model was trained to estimate the contribution of each subregion of the thalamus for the predictive model [47]. The steps of the calculation were similar with the GPC. After building linear kernels for subregions, these kernels and their associated labels were used to train the model. First, model parameters were estimated to define a decision function per kernel. The weight of each decision function was then estimated to provide a final decision function. The contribution of 


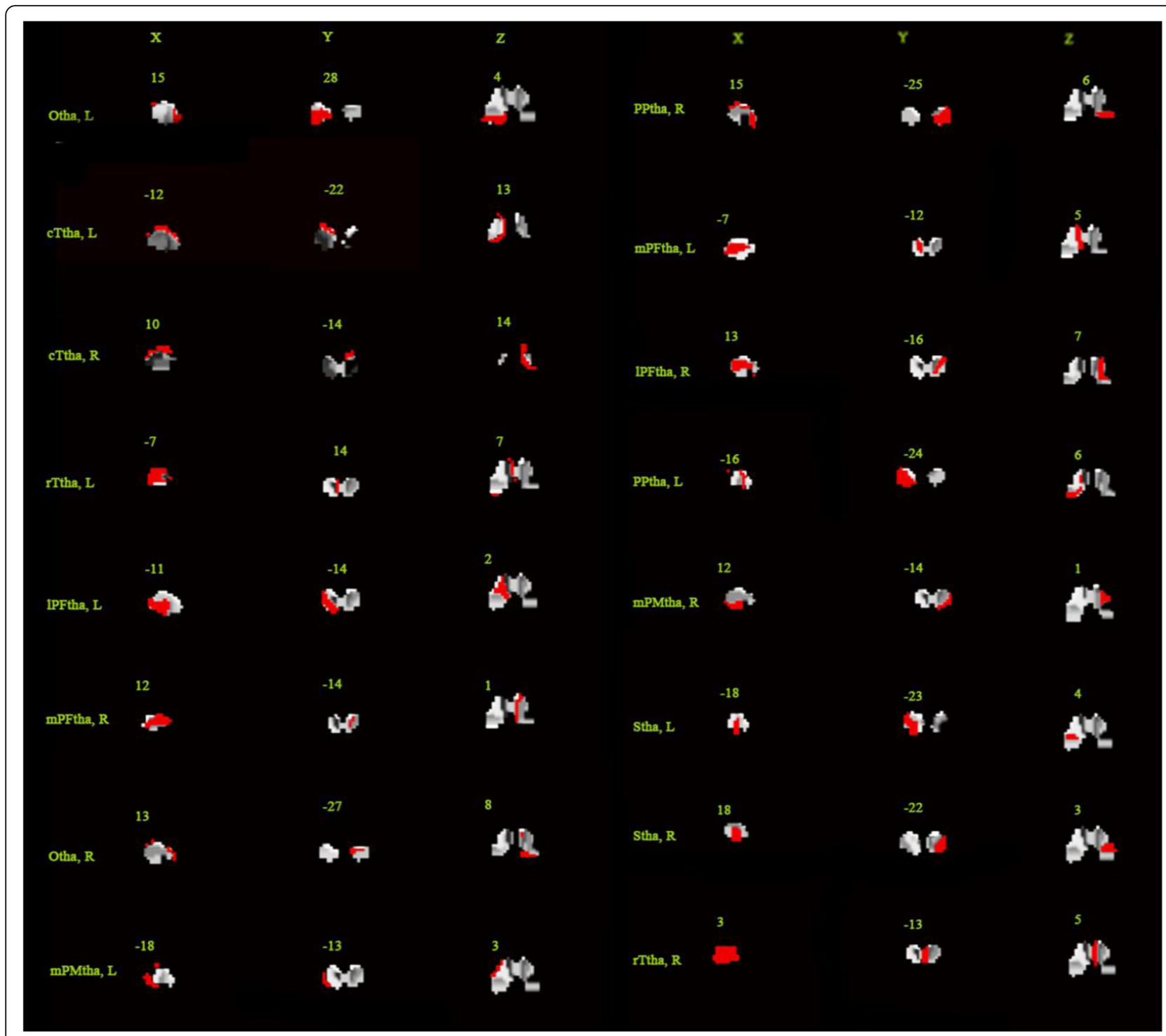

Fig. 1 Subregions of the thalamus. mPFtha, medial prefrontal thalamus; mPMtha, premotor thalamus; Stha, sensory thalamus; rTtha, rostral temporal thalamus; PPtha, posterior parietal thalamus; Otha, occipital thalamus; cTtha, caudal temporal thalamus; IPFtha, lateral prefrontal thalamus; L: left; R: right

each subregion for the decision function or predictive model can be explicitly computed [47]. We performed the MKL with the soft-margin parameters taking the default values $0.01,0.1,1,10$, and 100 . The evaluation of the model was the same as GPC and GPR.

\section{Results}

\section{Sample characteristics}

Table 1 shows the demographic variables and clinical characteristics of the two groups. Age $(Z=-0.83, P=$ $.410)$ and sex $\left(\chi^{2}=0.08, P=.776\right)$ in the MDD group and the $\mathrm{HC}$ group were well matched, and there was no significant difference between them according to the Mann-Whitney test and chi-square test, respectively.
Table 1 Demographic and Clinical Characteristics of Subjects

\begin{tabular}{lllll}
\hline Characteristic & MDD $(\boldsymbol{n}=\mathbf{7 4})$ & HC $(\boldsymbol{n}=\mathbf{4 4})$ & Statistic & $P$-Value \\
\hline Age (Years) & $26.53 \pm 8.56$ & $29.34 \pm 12.42$ & $Z=-0.83$ & .410 \\
Sex, n (\%) & & & & \\
$\quad$ Female & $49(66.22)$ & $28(63.64)$ & $X^{2}=0.08$ & .776 \\
$\quad$ Male & $25(33.78)$ & $16(36.36)$ & & \\
Education level & $4.68 \pm 0.74$ & $5.43 \pm 0.73$ & $X^{2}=39.24$ & $<.001$ \\
HAMD-24 score & $28.42 \pm 6.22$ & $1.36 \pm 1.37$ & $\mathrm{t}=36.01$ & $<.001$
\end{tabular}

$M D D$ major depressive disorder group, $H C$ healthy control group

Education level: 1 (illiterate), 2 (primary school), 3 (junior high school), 4

(senior high school), 5 (college or university), 6 (master's degree), 7 (doctorate) HAMD-24 Hamilton Depression Scale, 24-Item version 
Table 2 Clusters in the thalamus with abnormal GMD in the MDD patients relative to the healthy controls

\begin{tabular}{|c|c|c|c|c|c|c|c|c|}
\hline \multirow{2}{*}{$\begin{array}{l}\text { Cluster } \\
\text { location }\end{array}$} & \multirow{2}{*}{$\begin{array}{l}\text { Cluster } \\
\text { size }\end{array}$} & \multicolumn{3}{|l|}{ MNI } & \multicolumn{2}{|l|}{ GMD } & \multirow{2}{*}{$\begin{array}{l}\mathrm{T} \\
\text { value }\end{array}$} & \multirow[t]{2}{*}{$P$-Value } \\
\hline & & $x$ & $y$ & z & MDD & $\mathrm{HC}$ & & \\
\hline rTtha (L) & 9 & -9 & 1.5 & 6 & $0.55 \pm 0.04$ & $0.47 \pm 0.04$ & 9.80 & $<0.0001$ \\
\hline Otha (R) & 4 & 25.5 & -33 & 0 & $0.38 \pm 0.06$ & $0.55 \pm 0.08$ & -12.52 & $<0.0001$ \\
\hline Stha (R) & 2 & 16.5 & -24 & 3 & $0.35 \pm 0.02$ & $0.41 \pm 0.02$ & -17.85 & $<0.0001$ \\
\hline
\end{tabular}

MDD major depressive disorder group, HC healthy control group, GMD gray matter density, rTtha rostral temporal thalamus, Otha occipital thalamus, Stha sensory thalamus, $L$ left, $R$ right

Because the level of education was significantly higher in the HC group than in the MDD group, which may have potential effects on the results, the level of education was used as an influencing factor for the covariate analysis in all subsequent steps. HAMD-24 scores were also significantly higher in the patient group than in the $\mathrm{HC}$ group.

\section{Group-level results}

Structural differences between MDD participants and HCs Both GMD and GMV in the thalamus in MDD participants were significantly different from those in HC subjects. MDD patients were confirmed to have higher GMD in the left rostral temporal thalamus and lower GMD in the right occipital thalamus and sensory thalamus than HC subjects; MDD patients exhibited higher GMV in the left lateral prefrontal thalamus, the right posterior parietal thalamus, and the right rostral temporal thalamus and lower GMV in the right medial prefrontal thalamus, the right sensory thalamus, and the left rostral temporal thalamus than HCs (see Table 2, Table 3 and Fig. 2a).

\section{Rs-fMRI differences between MDD patients and HCs}

No clusters verified significant differences between MDD patients and HCs in ALFF or fALFF in the thalamus, as shown in Fig. $2 \mathrm{~b}$.

\section{Individual-level prediction: MDD vs. HC participants GPCs trained with sMRI features}

The GMD and GMV of the thalamus were used to train the GPC. The accuracy of GPC based on GMD of the thalamus was $96.59 \%(P<.001)$, the sensitivity was
$100 \%$, and the specificity was $93.18 \%$. The accuracy of GPC trained with thalamic GMV was $93.18 \%(P<.001)$, and the sensitivity and specificity were $100 \%$ and $86.36 \%$, respectively (see Table 4 and Fig. 3 ).

\section{MKLs trained with sMRI features}

The accuracy of MKL based on GMD of the thalamus was $97.73 \%(P<.001)$, the sensitivity was $100 \%$, and the specificity was $95.45 \%$. The accuracy of MKL trained with thalamic GMV was $98.86 \%(P<.001)$, and the sensitivity and specificity were 100 and $97.73 \%$, respectively (see Table 5). The contribution of each subregion to the classification is shown in Table 6.

\section{GPCs trained with rs-fMRI features}

The accuracy of GPCs trained with ALFF and fALFF was at the chance level (see Table 7 and Fig. 4). The accuracy of GPC trained with ALFF in the thalamus was $40.54 \%(P=.808)$, and the accuracy of GPC trained with fALFF in the thalamus was $47.97 \%(P=.534)$.

\section{Individual-level prediction of HAMD scores GPRs trained with sMRI features}

This study built a GPR model that used gray matter information to predict the HAMD scores of participants. The correlation between HAMD score targets and predictions in the GPR trained with the GMD of the thalamus was 0.90 , the $P$-value was lower than 0.001 , and the coefficient of determination $r^{2}=0.82$. In a GPR model trained with the GMV of the thalamus, the correlation between HAMD score targets and predictions was 0.89 , the $P$-value was lower than 0.001 , and $r^{2}=$ 0.79 . Figure 5 shows the results.

Table 3 Clusters in the thalamus with abnormal GMV in the MDD patients relative to the healthy controls

\begin{tabular}{|c|c|c|c|c|c|c|c|c|}
\hline \multirow{2}{*}{$\begin{array}{l}\text { Cluster } \\
\text { location }\end{array}$} & \multirow{2}{*}{$\begin{array}{l}\text { Cluster } \\
\text { size }\end{array}$} & \multicolumn{3}{|l|}{ MNI } & \multicolumn{2}{|l|}{ GMV } & \multirow{2}{*}{$\begin{array}{l}\mathrm{T} \\
\text { value }\end{array}$} & \multirow{2}{*}{$\begin{array}{l}P \text { - } \\
\text { Value }\end{array}$} \\
\hline & & $x$ & $y$ & z & MDD & $\mathrm{HC}$ & & \\
\hline IPFtha (L) & 53 & -3 & -19.5 & -4.5 & $0.29 \pm 0.03$ & $0.27 \pm 0.02$ & 4.84 & $<.0001$ \\
\hline PPtha (R) & 12 & 9 & -31.5 & 0 & $0.32 \pm 0.05$ & $0.24 \pm 0.04$ & 9.99 & $<.0001$ \\
\hline mPFtha (R) & 12 & 6 & -4.5 & 0 & $0.19 \pm 0.03$ & $0.23 \pm 0.04$ & -6.55 & $<.0001$ \\
\hline Stha (R) & 31 & 16.5 & -24 & 4.5 & $0.27 \pm 0.02$ & $0.33 \pm 0.03$ & -12.24 & $<.0001$ \\
\hline rTtha (L) & 58 & -4.5 & 1.5 & 3 & $0.24 \pm 0.04$ & $0.22 \pm 0.03$ & 2.75 & .007 \\
\hline
\end{tabular}

$M D D$ major depressive disorder group, $H C$ healthy control group, GMV gray matter volume, IPFtha lateral prefrontal thalamus, PPtha posterior parietal thalamus, mPFtha medial prefrontal thalamus, Stha sensory thalamus, $r T$ tha rostral temporal thalamus, $L$ left, $R$ right 


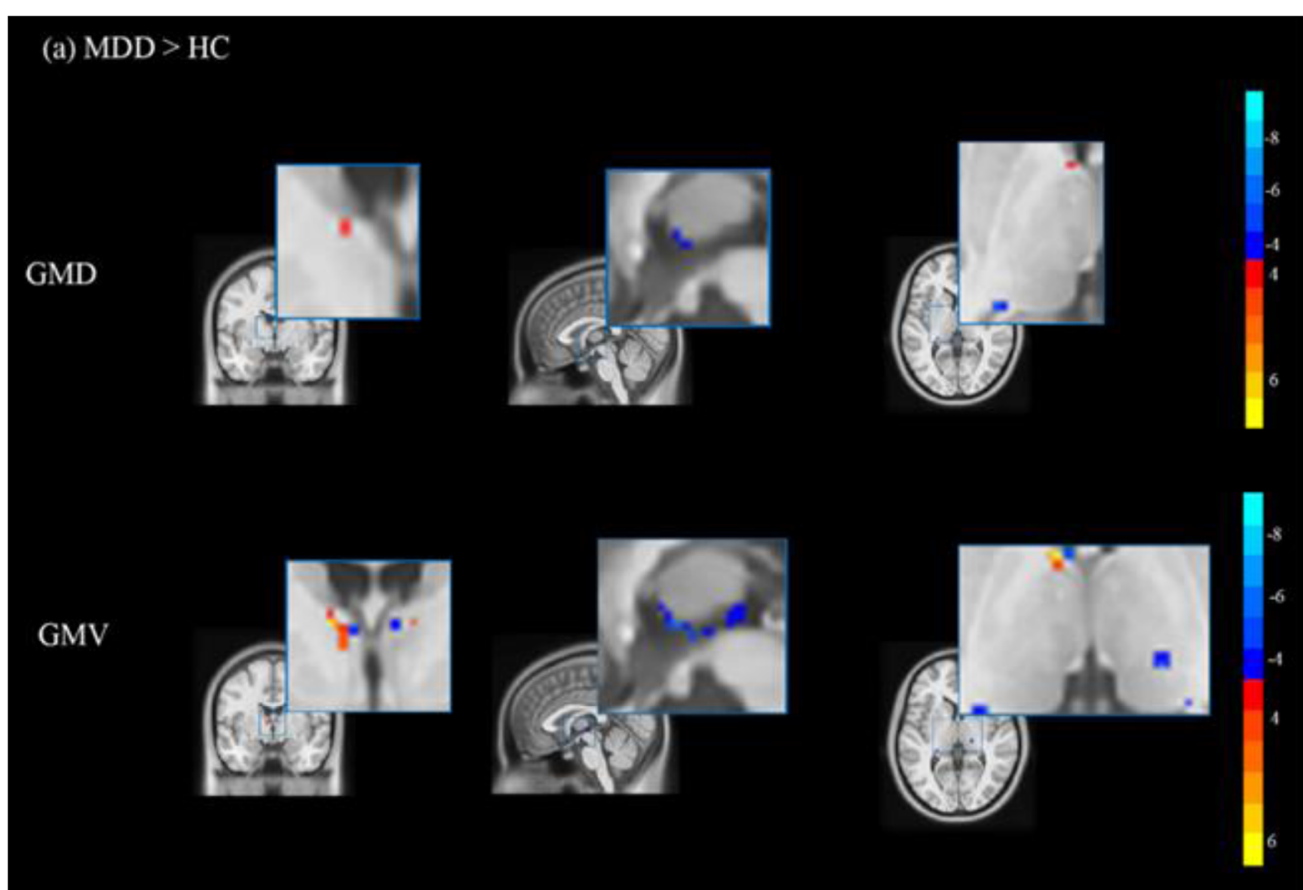

(b) $\mathrm{MDD}>\mathrm{HC}$

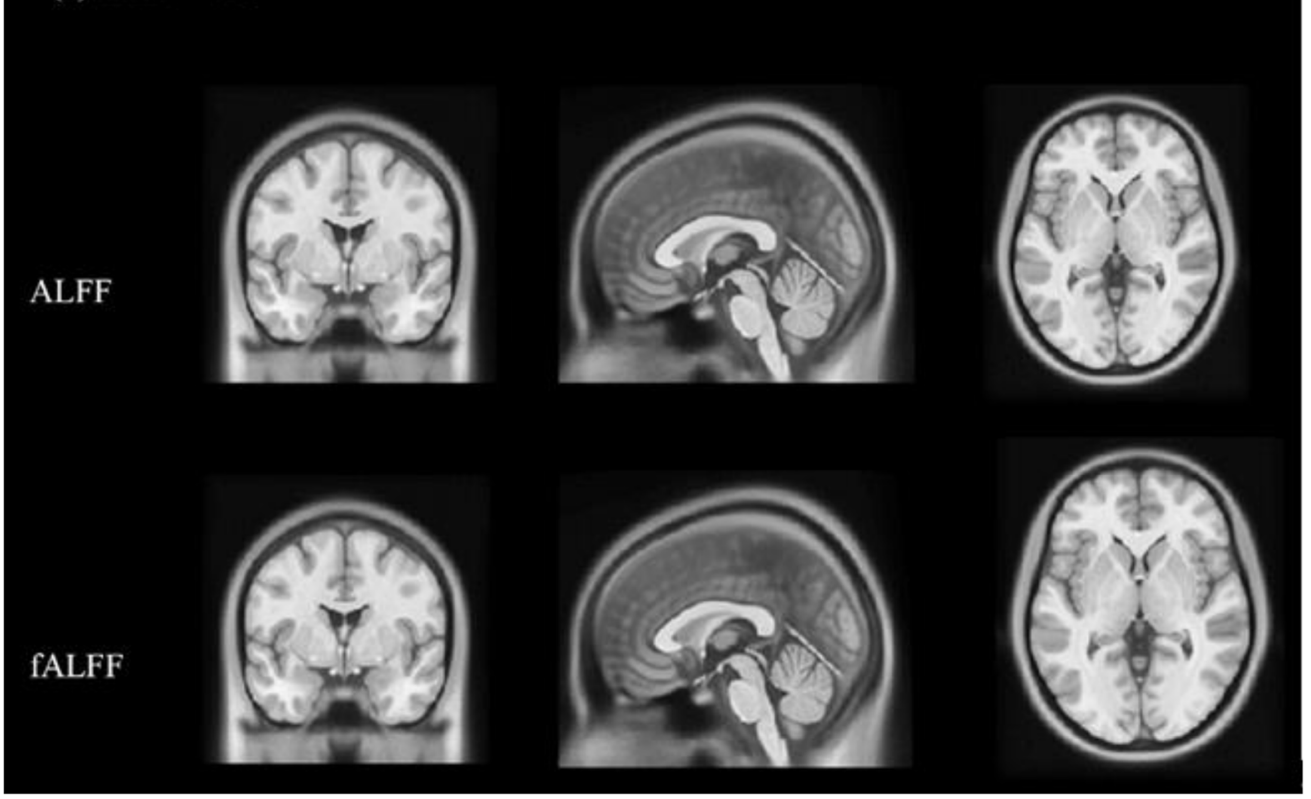

Fig. 2 (a). The picture shows significant differences between the MDD patients and HC participants in thalamic GMD and GMV. (b) The picture shows no significant differences between the MDD patients and HC participants in ALFF and fALFF in the thalamus (Gaussian random fieldcorrected, voxel $p$-value $=0.001$, cluster $p$-value $=0.05$ )

\section{GPRs trained with rs-fMRI features}

The GPR models trained with rs-fMRI data showed a negative correlation between the true HAMD scores and predictions, which implied that the GPR models cannot correctly predict the HAMD scores. In the GPR trained with ALFF in the thalamus, the correlation between HAMD score targets and predictions was $-0.92(P=.640$, $\left.r^{2}=0.84\right)$. The correlation between targets and predictions in the GPR trained with fALFF of the thalamus was -0.92 $\left(P=.872, r^{2}=0.84\right)$. The results are shown in Fig. 6 .

\section{Discussion}

In this study, we investigated the potential capacity of the two structural features (i.e., GMD and GMV) and 
Table 4 The performance of GPCs trained with GMD and GMV

\begin{tabular}{lllll}
\hline Indicators & Balanced accuracy (\%) & BA p-value & Sensitivity (\%) & Specificity (\%) \\
\hline GMD & 96.59 & $<0.001$ & 100.00 & 93.18 \\
GMV & 93.18 & $<0.001$ & 100.00 & 86.36 \\
\hline
\end{tabular}

GPC Gaussian process classification, GMD gray matter density, GMV gray matter volume

the two rs-fMRI features (i.e., ALFF and fALFF) of the thalamus in the diagnosis of MDD at the individual level by MVPA methods (i.e., GPC and GPR). The results showed that the balanced accuracy of the machine learning models trained with thalamic GMD and GMV was significantly higher than the chance level. The correlation between the real and the predicted HAMD scores in the GPRs trained with GMD and GMV was significant. The results also showed that the models trained with ALFF and AALFF in the thalamus failed to discriminate MDD patients from $\mathrm{HC}$ participants. Findings from this study suggest that the structural MRI features rather than the rs-fMRI features of the thalamus may have good potentials for the individualized diagnosis of MDD.

This study confirms that the thalamus is closely related to MDD, and different machine learning models (i.e., GPC and GPR) trained with thalamic gray matter imaging indicators showed good performance in identifying MDD patients, which corresponded with our group-level results showing significantly different clusters in the thalamus. It is well known that all sensory nerve pathways, except for those conveying olfactory information, project to the thalamus [67]. In other words, the thalamus is a sensory relay station that is involved in emotion, memory, and arousal [11]. Some evidence has been illustrated to support the argument that the thalamus is not simply a relay station [13] but also plays a central role in ongoing cortical functioning [12]. The thalamus is globally connected with distributed cortical regions, most thalamic subdivisions display network properties that are capable of integrating multimodal information across diverse cortical functional networks, and the thalamus is involved in multiple cognitive functions [13]. Additionally, evidence has suggested that the human thalamus is a critical hub region that could integrate diverse information being processed throughout the cerebral cortex [13]. The thalamus relays this information to the corresponding cerebral cortical areas and from there to the amygdala and hippocampus, which are the regions of the brain most closely related to emotion, memory, and arousal [11]. Depressed mood, loss of energy, difficulty with short-term memory, etc. are included in the core symptoms of a depressive episode
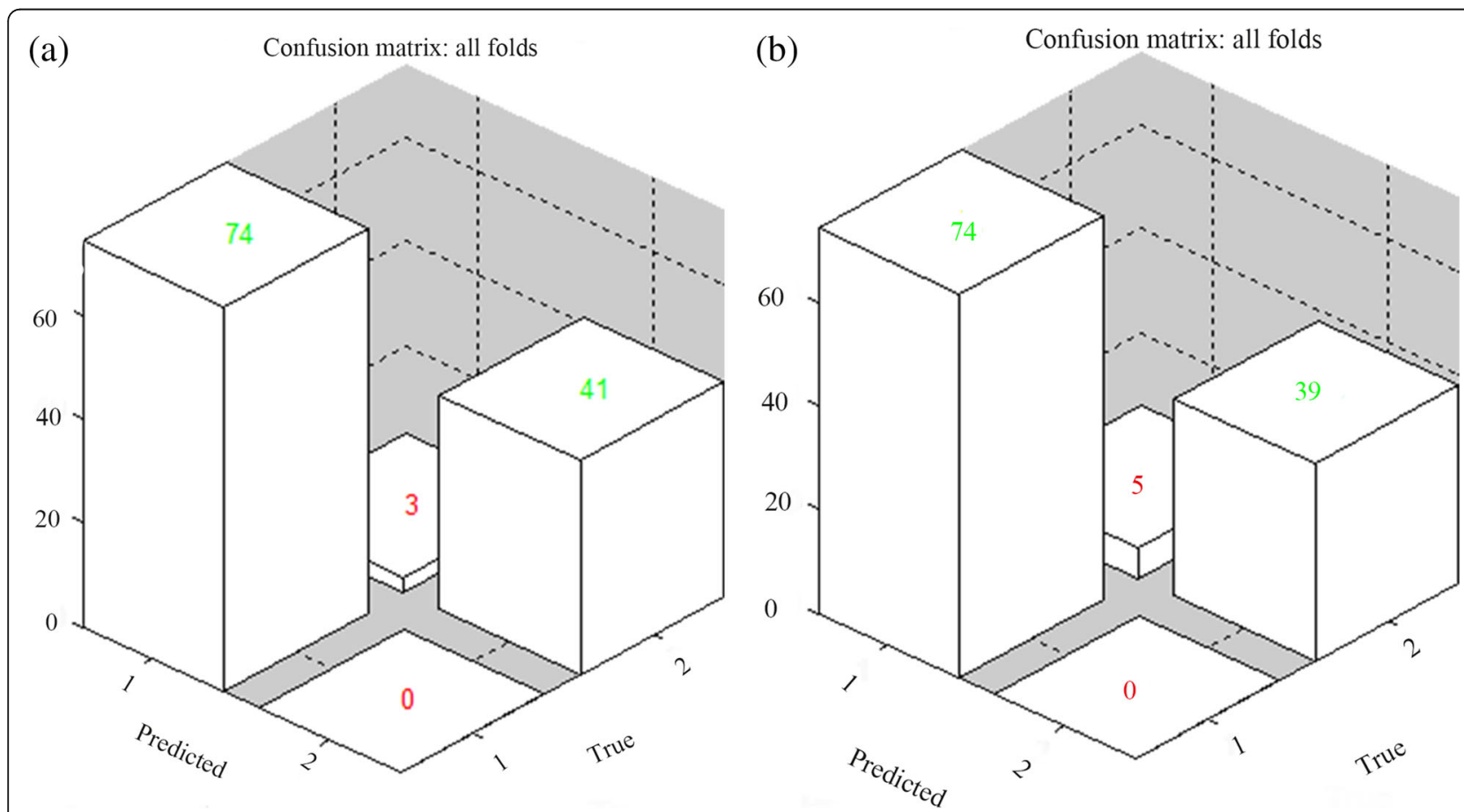

Fig. 3 The left image (a) shows the classification performance using gray matter density (GMD) data in the thalamus: the balanced accuracy was $96.59 \%$, the sensitivity was $100 \%$, and the specificity was $93.18 \%$. The right image (b) shows the classification performance using gray matter volume (GMV) data in the thalamus: the balanced accuracy was $93.18 \%$, the sensitivity was $100 \%$, and the specificity was $86.36 \%$ 
Table 5 The performance of MKLs trained with GMD and GMV

\begin{tabular}{lllll}
\hline Indicators & Balanced accuracy (\%) & BA p-value & Sensitivity (\%) & Specificity (\%) \\
\hline GMD & 97.73 & $<0.001$ & 100.00 & 95.45 \\
GMV & 98.86 & $<0.001$ & 100.00 & 97.73 \\
\hline
\end{tabular}

MKL Multiple Kernel Learning, GMD gray matter density, GMV gray matter volume

[68]. Furthermore, a recent review of previous studies confirmed that the results of rodent studies indicate that thalamocortical circuits are candidates for controlling the activity of the default network, including tasksuppression effects [27]. Dysregulation of thalamocortical circuits might also increase the risk of certain forms of mental illness [27]. MRI studies have demonstrated that MDD patients have abnormalities in prefrontal, temporal, parietal, insular, occipital, and subcortical structures $[54,55]$. The abovementioned brain areas are all related to thalamocortical circuits. If the gray matter in the thalamus, an important part of thalamocortical circuits, is abnormal, it may cause the whole thalamocortical circuit to be abnormal, which may lead to MDD [27]. Therefore, the analysis of structural imaging data from the thalamus could distinguish MDD patients from healthy people. Such an analysis, when performed at the individual level by employing MVPA as in our study, is more valuable for predicting individual cases.

The group-level analysis of the gray matter features of the thalamus reported significantly different clusters

Table 6 The weights of thalamic subregions for MKLS

\begin{tabular}{|c|c|c|c|}
\hline \multicolumn{2}{|l|}{ GMD } & \multicolumn{2}{|l|}{ GMV } \\
\hline Location & Weights (\%) & Location & Weights (\%) \\
\hline IPFtha, L & 32.87 & IPFtha, L & 35.31 \\
\hline cTtha, R & 23.53 & cTtha, R & 28.84 \\
\hline Stha, R & 12.97 & Stha, $R$ & 13.29 \\
\hline mPMtha, R & 11.28 & rTtha, L & 12.56 \\
\hline rTtha, L & 9.97 & mPMtha, L & 4.29 \\
\hline mPMtha, L & 7.21 & Otha, R & 3.40 \\
\hline rTtha, $R$ & 2.18 & mPMtha, R & 1.81 \\
\hline mPFtha, L & 0.00 & Stha, L & 0.26 \\
\hline mPFtha, R & 0.00 & Otha, L & 0.24 \\
\hline Stha, L & 0.00 & mPFtha, L & 0.00 \\
\hline PPtha, L & 0.00 & mPFtha, $R$ & 0.00 \\
\hline PPtha, R & 0.00 & rTtha, R & 0.00 \\
\hline Otha, L & 0.00 & PPtha, L & 0.00 \\
\hline Otha, R & 0.00 & PPtha, $\mathrm{R}$ & 0.00 \\
\hline cTtha, L & 0.00 & cTtha, L & 0.00 \\
\hline IPFtha, R & 0.00 & IPFtha, R & 0.00 \\
\hline
\end{tabular}

MKL Multiple Kernel Learning, GMD gray matter density, GMV gray matter volume, mPFtha medial prefrontal thalamus, mPMtha premotor thalamus, Stha sensory thalamus, rTtha rostral temporal thalamus, PPtha posterior parietal thalamus, Otha occipital thalamus, cTtha caudal temporal thalamus, IPFtha lateral prefrontal thalamus, $L$ left, $R$ right between the MDD patients and HCs. Concurrently, the subregions where most of the clusters are located had high contribution weights in the classification. For the first time, we found that heterogeneity in the thalamus at the subregional level identified individuals with depression. The thalamus comprises numerous nuclei, which project to different brain areas and receive inputs from other cortical or subcortical brain regions $[11,13]$. The difference in connections between the different thalamic subregions and other brain regions may be associated with different functions in thalamic subregions. The medial dorsal nucleus of the thalamus may play a role in memory (perhaps specifically in the retrieval of episodic memory), mood, motivation, and the sleep/wake cycle [11]. The anterior nucleus of the thalamus may be involved in memory, modulation of the sleep/wake cycle, and directed attention [11]. The lateral dorsal nucleus of the thalamus may be related to motivation and/or attention with sensory processes [11]. Thus, this heterogeneity may explain the differences in the results across thalamic subregions. The findings of heterogeneity across thalamic subregions were indirectly supported by the results of our previous study [6], which, through functional imaging data, revealed that MDD patients exhibited distinct resting-state functional connectivity patterns across thalamic subregions.

Results from this study suggest that ALFF and fALFF in the thalamus may not be robust features for recognizing MDD patients. No significant difference in ALFF or fALFF clusters in the thalamus was found between the MDD and HC groups, and machine learning models trained with ALFF and AALFF in the thalamus failed to effectively discriminate individual patients from healthy people with ideal performance in this study. We also performed a two-sample t-test on the whole brain and found that the differences of ALFF and AALFF were located in the cortex area and brainstem, not the thalamus (see supplementary materials Fig. S1). ALFF, in which the square root of the power spectrum was integrated in a low-frequency range, was used to detect the regional intensity of spontaneous fluctuations in the BOLD signal [69]. In fALFF, the ratio of the power spectrum of the low-frequency $(0.01-0.08 \mathrm{~Hz})$ range to that of the entire frequency range was computed [60]. Most neuroimaging MDD studies have reported abnormal ALFF and fALFF levels in the left cerebellum, amygdala, left hippocampus, precuneus, right cingulate cortex, right putamen, medial prefrontal cortex, left motor cortex and parietal lobe 
Table 7 The performance of GPCS trained with ALFF and fALFF

\begin{tabular}{lllll}
\hline Indicators & Balanced accuracy (\%) & BA p-value & Sensitivity (\%) & Specificity (\%) \\
\hline ALFF & 40.54 & 0.808 & 81.08 & 0.00 \\
fALFF & 47.97 & 0.534 & 95.95 & 0.00 \\
\hline
\end{tabular}

GPC Gaussian process classification, ALFF amplitude of low-frequency fluctuations, fALFF fractional amplitude of low-frequency fluctuations

[56-59], and some researches have found that abnormal thalamic ALFF or fALFF may correlate with the antidepressant response but not MDD onset [9]. Another reason why this study showed negative ALFF and fALFF results in the thalamus was that a few days before enrollment some of the MDD patients were taking antidepressants, which may have influenced the research results. Some studies have reported that ALFF and fALFF could be changed by antidepressant use [70]. Thus, the reason why the ALFF and AALFF features of the thalamus did not have good enough performance in discriminating MDD individuals in our study may in part be related to this.

To our knowledge, this is the first study to focus on the thalamus for the individualized diagnosis of MDD. Using machine learning methods to analyze the MRI data of thalamus, this study established an individualized brain morphology-related diagnostic model for MDD based on thalamic imaging features. If this model could be applied in clinic, it is expected to be helpful to improve the current situation that the diagnosis of MDD in psychiatric clinic depends mainly on patients' selfstatement and psychiatrists' subjective judgment, and also helpful to reduce the risk of misdiagnosis of MDD. Further, the results of this study not only may provide an important basis for the early identification and objective diagnosis of MDD at individual level, but also may provide useful clues for the exploration of the biological and pathological mechanism behind MDD. Additionally, our study reveals for the first time the heterogeneity in the structural features of thalamic subregions for predicting MDD at the individual level, which demonstrates the importance and the heterogeneity of the thalamus in MDD, and may provide some clue for further research about the whole thalamus and thalamic subregions in emotion-related disorders.

There are some limitations in this study: [1] Although 118 subjects were included in this study, which exceeded the sample size of most previous single-center studies, the sample size of this study was still not large enough

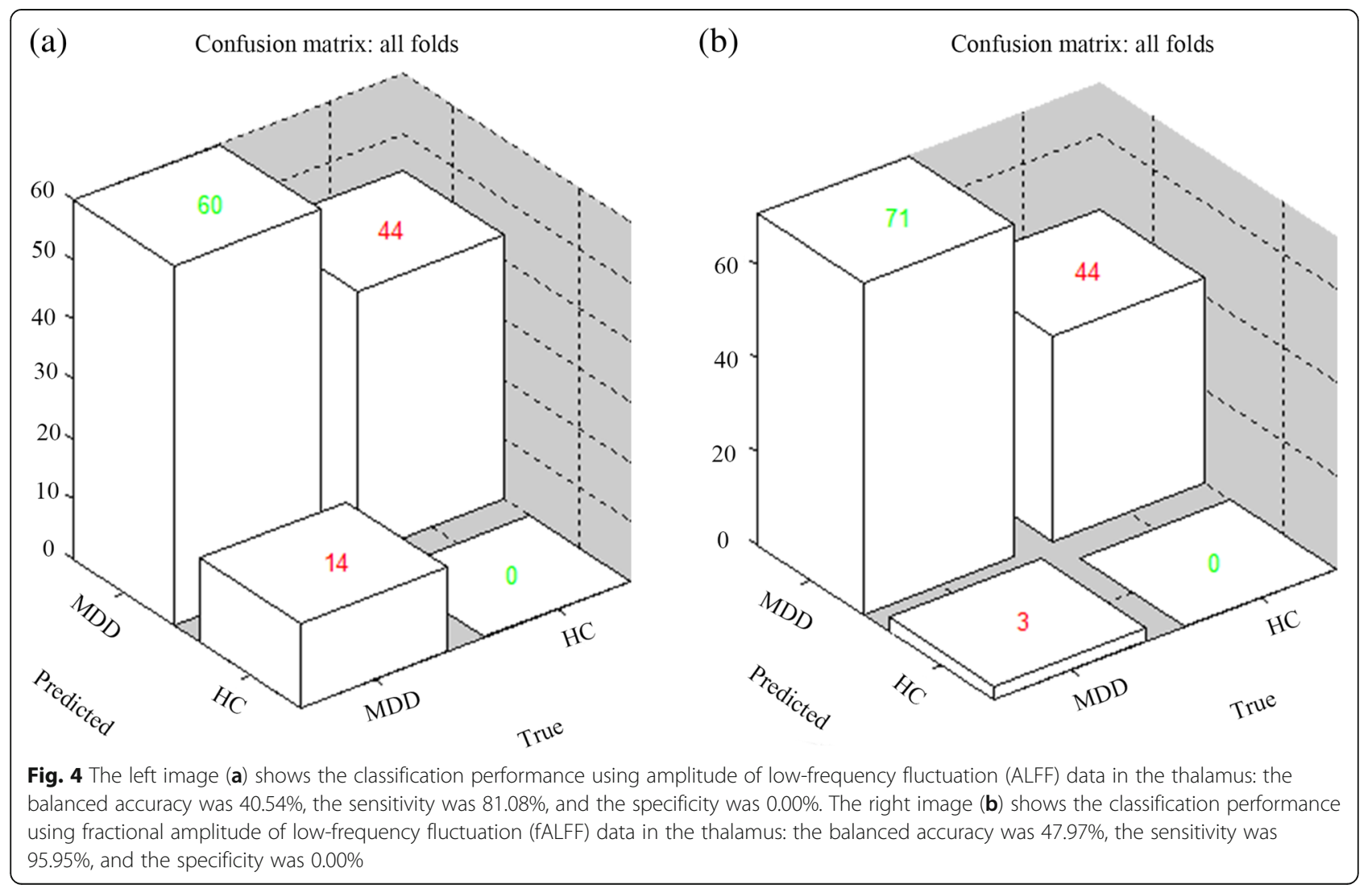


(a)

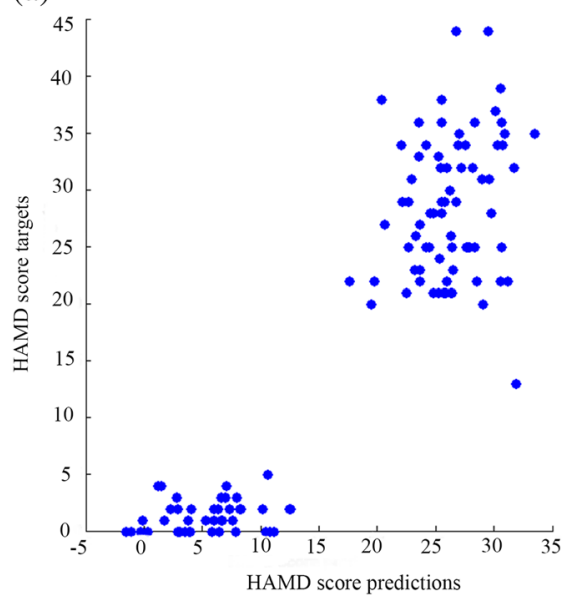

(b)

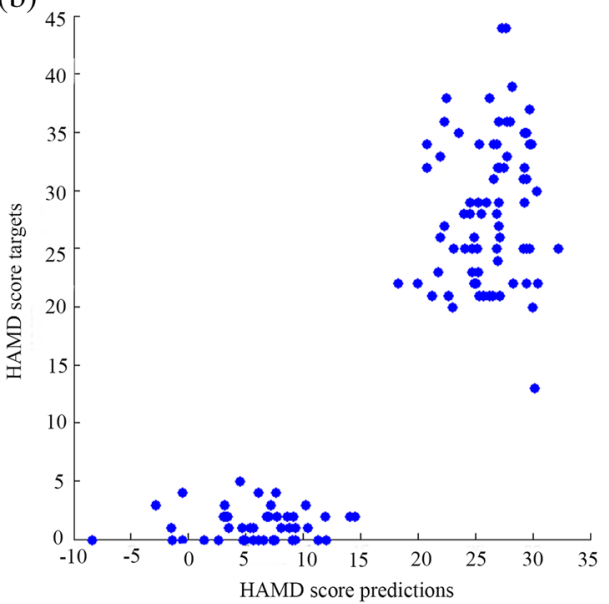

Fig. 5 HAMD score targets and HAMD score predictions. "HAMD score targets" means "the actual HAMD score". The left graph (a) shows the performance of the GPR trained with the GMD in the thalamus, and the right graph (b) shows the performance of the GPR trained with the GMV in the thalamus

(from the perspective of requirements of multivariate pattern analysis method), which may lead to some deviation between the classification results and the actual situation. We know that small data sets may possibly lead to overfitting. By building a larger database upon which to base a predictive model, the variations observed among MDD patients could be more thoroughly incorporated, which, in the future, may result in models with better clinical utility [71]. In future studies, it will be necessary to explore the validation of the performance of GPC and GPR in an independent large database [2]. Not all MDD patients in our study were medication-free subjects, and some of them were not in their first depressive episode. This may have had some influence on the results of this study. These problems need to be addressed in future studies [3]. In this study, the machine learning models trained with ALFF and fALFF in the thalamus failed to effectively discriminate individual MDD patients from healthy persons. This definitely does not mean that there are no functional

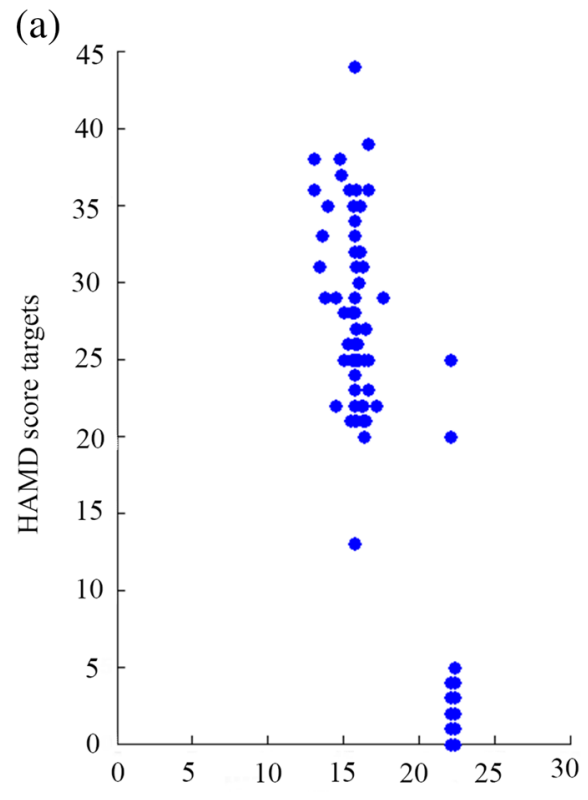

HAMD score predictions

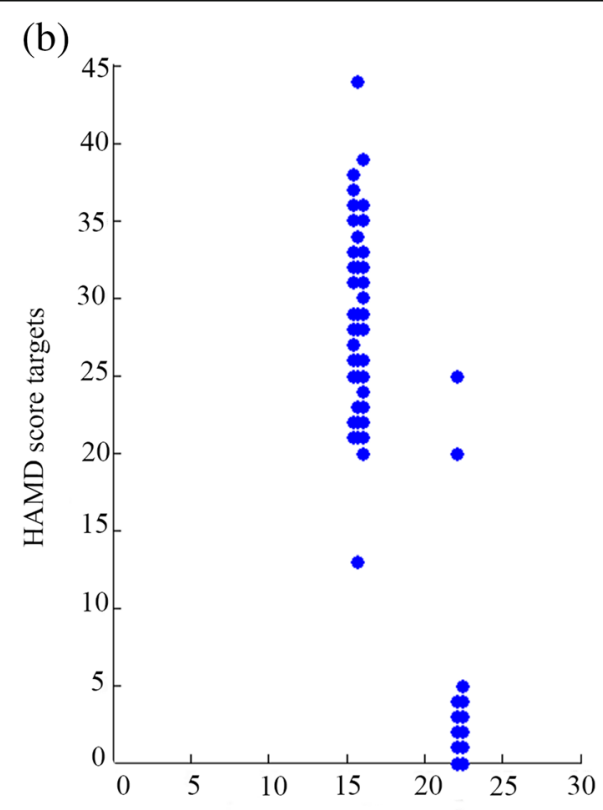

HAMD score predictions

Fig. 6 The left graph (a) shows the performance of the GPR trained with ALFF in the thalamus, and the right graph (b) shows the performance of the GPR trained with fALFF in the thalamus 
alterations in the thalamus in MDD. Nor does it mean that other functional MRI data of the thalamus are not suitable to be chosen as features for individualized recognition of MDD patients. One of our previous studies showed sample entropy changes in the bilateral thalami in MDD patients [6], so we are considering using the sample entropy of the resting-state fMRI data as a feature for the classification and prediction of MDD in our future studies [4]. The whole brain volume was not controlled as a covariate in this study, which may have potential correlation with the volume of the thalamus, and could be considered in future studies.

\section{Conclusions}

In conclusion, this is the first study to focus on the thalamus and to use machine learning methods to differentiate MDD patients from healthy people. Both classifiers trained with gray matter volume data and gray matter density data have been confirmed to have high discriminatory accuracy by pattern analysis. Both GPRs trained with the GMD and the GMV in the thalamus could predict HAMD scores of the participants. The GPCs and GPRs trained with ALFF and fALFF in the thalamus showed poor performance in recognizing MDD patients. Therefore, the results of this study suggest that gray matter information, but not functional information, in the thalamus has good potential for the individualized diagnosis of MDD. It would be expected that our results would not only provide important basis for the early identification and objective diagnosis of MDD, but also provide useful clues for the exploration of the biological and pathological mechanism behind MDD.

\footnotetext{
Abbreviations

MRI: Magnetic resonance imaging; MDD: Major depressive disorder; HCs: Healthy controls; GMD: Gray matter density; GMV: Gray matter volume; ALFF: Amplitude of low-frequency fluctuations; fALFF: Fractional amplitude of low-frequency fluctuations; GPR: Gaussian process regression; GPC: Gaussian process classifier; MVPA: Multivariate pattern analysis; GLM: General linear model; SVM: Support vector machine; BD: Bipolar disorder; sMRI: Structural magnetic resonance imaging; rs-fMRl: Resting-state functional magnetic resonance imaging; CCBD: Center for Cognition and Brain Disorders; HZNU: Hangzhou Normal University; MNI: Montreal Neurological Institute; WM: White matter; CSF: Cerebrospinal fluid; mPFtha: Medial prefrontal thalamus; mPMtha: Premotor thalamus; Stha: Sensory thalamus; rTtha: Rostral temporal thalamus; PPtha: Posterior parietal thalamus; Otha: Occipital thalamus; cTtha: Caudal temporal thalamus; IPFtha: Lateral prefrontal thalamus; L: Left; R: Right
}

\section{Supplementary Information}

The online version contains supplementary material available at https://doi. org/10.1186/s12888-021-03414-9.

\section{Additional file 1.}

\section{Acknowledgments}

This study was funded by the National Natural Science Foundation of China (81271503). We thank Xiaole Wang and Xiaojiao Hu for their participation in the early stage of data collection and thank Xiaolong Li for providing technical support for data analysis in this study.

\section{Authors' contributions}

Donglin Wang originated the study and revised the manuscript. Sutao Song supervised the data processing and revised the manuscript. Hanxiaoran Li conducted the statistical analyses and drafted the manuscript. Zhonglin Tan, Zhenzhen Lian, Yan Wang, Xin Zhou, and Chenyuan Pan collected the relevant data. All authors have read and agree with the contents of the manuscript, and there are no financial interests to report.

\section{Funding}

This study was funded by the National Natural Science Foundation of China (81271503)

\section{Availability of data and materials}

The datasets used and/or analyzed during the current study are available from the corresponding author on reasonable request.

\section{Declarations}

\section{Ethics approval and consent to participate}

This study was approved by the ethics committee of the Institutes of Psychological Sciences, Hangzhou Normal University. All methods were performed in accordance with the relevant guidelines and regulations. All patients' legally authorized representatives and controls provided written informed consent before participation in the study procedures.

\section{Consent for publication}

Not applicable.

\section{Competing interests}

The authors declare no conflicts of interest.

\section{Author details}

${ }^{1}$ Institutes of Psychological Sciences, College of Education, Hangzhou Normal University, 2318\#, Yuhangtang Rd, Hangzhou 311121, China. ${ }^{2}$ Center for Cognition and Brain Disorders, Hangzhou Normal University, Hangzhou 311121, China. ${ }^{3}$ Zhejiang Key Laboratory for Research in Assessment of Cognitive Impairments, Hangzhou 311121, China. ${ }^{4}$ School of Information Science and Engineering, Shandong Normal University, 1\#, University Rd, Changqing District, Jinan 250358, China. ${ }^{5}$ Department of Psychiatry, The Affiliated Hospital, Hangzhou Normal University, Hangzhou 310015, China. ${ }^{6}$ Department of Psychiatry, Hangzhou Seventh People's Hospital, Hangzhou 310013, China.

Received: 9 December 2020 Accepted: 4 August 2021

Published online: 20 August 2021

\section{References}

1. Dunner DL. Major depressive disorder. Med Basis Psychiatry Third Ed. 2008: 73-83. https://doi.org/10.1007/978-1-59745-252-6_5.

2. Bilello JA. Seeking an objective diagnosis of depression. Biomark Med. 2016; 10(8):861-75. https://doi.org/10.2217/bmm-2016-0076.

3. Busatto GF. Structural and functional neuroimaging studies in major depressive disorder with psychotic features: a critical review. Schizophr Bull. 2013;39(4):776-86. https://doi.org/10.1093/schbul/sbt054.

4. Graham J, Salimi-Khorshidi G, Hagan C, Walsh N, Goodyer I, Lennox B, et al. Meta-analytic evidence for neuroimaging models of depression: state or trait? J Affect Disord. 2013;151(2):423-31. https://doi.org/10.1016/j.jad.2013. 07.002 .

5. Depeursinge A, Racoceanu D, lavindrasana J, et al. Fusing Visual and Clinical Information for Lung Tissue Classification in HRCT Data. Artif Intell Med. 2010;229:ARTMED1118. https://doi.org/10.1016/j.artmed.2010.04.006.

6. Xue SW, Wang D, Tan Z, Wang Y, Lian Z, Sun Y, et al. Disrupted brain entropy and functional connectivity patterns of thalamic subregions in major depressive disorder. Neuropsychiatr Dis Treat. 2019;15:2629-38. https://doi.org/10.2147/NDT.S220743.

7. Hahn T, Marquand AF, Ehlis AC, Dresler T, Kittel-Schneider S, Jarczok TA, et al. Integrating neurobiological markers of depression. Arch Gen 
Psychiatry. 2011;68(4):361-8. https://doi.org/10.1001/archgenpsychiatry.201 0.178 .

8. Lopez-Larson M, King JB, McGlade E, et al. Enlarged thalamic volumes and increased fractional anisotropy in the thalamic radiations in veterans with suicide behaviors. Front Psychiatry. 2013;4(AUG):1-13. https://doi.org/10.33 89/fpsyt.2013.00083.

9. Yamamura T, Okamoto Y, Okada G, et al. Association of thalamic hyperactivity with treatment-resistant depression and poorresponse in early treatment for major depression: A resting-state fMRI study using fractional amplitude of low-frequencyfluctuations. Transl Psychiatry. 2016;6(3). https:// doi.org/10.1038/tp.2016.18

10. Yuan R, Di X, Taylor PA, Gohel S, Tsai YH, Biswal BB. Functional topography of the thalamocortical system in human. Brain Struct Funct. 2016;221(4): 1971-84. https://doi.org/10.1007/s00429-015-1018-7.

11. Taber KH, Wen C, Khan A, Hurley RA. The limbic thalamus. J Neuropsychiatry Clin Neurosci. 2004;16(2):127-32. https://doi.org/10.1176/appi.neuropsych.1 6.2.127.

12. Sherman SM. Thalamus plays a central role in ongoing cortical functioning. Nat Neurosci. 2016;19(4):533-41. https://doi.org/10.1038/nn.4269.

13. Hwang K, Bertolero MA, Liu WB, D'Esposito M. The human thalamus is an integrative hub for functional brain networks. J Neurosci. 2017;37(23):5594607. https://doi.org/10.1523/JNEUROSCI.0067-17.2017.

14. Peters SK, Dunlop K, Downar J. Cortico-striatal-thalamic loop circuits of the salience network: A central pathway in psychiatric disease and treatment. Front Syst Neurosci. 2016;10(DEC):1-23. https://doi.org/10.3389/fnsys.2016. 00104.

15. Deng $F$, Wang $Y$, Huang $H$, Niu M, Zhong $S$, Zhao L, et al. Abnormal segments of right uncinate fasciculus and left anterior thalamic radiation in major and bipolar depression. Prog Neuro-Psychopharmacology Biol Psychiatry. 2018;81:340-9. https://doi.org/10.1016/j.pnpbp.2017.09.006.

16. Zhao YJ, Du MY, Huang XQ, et al. Brain grey matter abnormalities in medicationfree patients with major depressive disorder: a meta-analysis. Psychol Med. 2014; 44(14):2927-37. https://doi.org/10.1017/S0033291714000518.

17. Jenkins L, Chiang J, Alpert K, Wang L, Miller G. F53. Outward Subcortical Deformations Associated With Sub-Clinical Depression Symptoms in Adolescents. Biol Psychiatry. 2018;83(9):S258. https://doi.org/10.1016/j. biopsych.2018.02.666.

18. Lu Y, Liang H, Han D, Mo Y, Li Z, Cheng Y, et al. The volumetric and shape changes of the putamen and thalamus in first episode, untreated major depressive disorder. Neurolmage Clin. 2016;11:658-66. https://doi.org/10.101 6/j.nicl.2016.04.008.

19. Zhang X, Yao S, Zhu X, Wang X, Zhu X, Zhong M. Gray matter volume abnormalities in individuals with cognitive vulnerability to depression: a voxel-based morphometry study. J Affect Disord. 2012;136(3):443-52. https://doi.org/10.1016/j.jad.2011.11.005

20. Nugent AC, Davis RM, Zarate CA, Drevets WC. Reduced thalamic volumes in major depressive disorder. Psychiatry Res Neuroimaging. 2013;213(3):179-85. https://doi.org/10.1016/j.pscychresns.2013.05.004.

21. Ahn SJ, Kyeong S, Suh SH, Kim JJ, Chung TS, Seok JH. What is the impact of child abuse on gray matter abnormalities in individuals with major depressive disorder: a case control study. BMC Psychiatry. 2016;16(1):1-7. https://doi.org/10.1186/s12888-016-1116-y.

22. Hagan CC, Graham JME, Tait R, Widmer B, van Nieuwenhuizen AO, Ooi C, et al. Adolescents with current major depressive disorder show dissimilar patterns of age-related differences in ACC and thalamus. Neurolmage Clin. 2015;7:391-9. https://doi.org/10.1016/j.nicl.2014.12.019.

23. Bora E, Harrison BJ, Davey CG, Yücel M, Pantelis C. Meta-analysis of volumetric abnormalities in cortico-striatal-pallidal- thalamic circuits in major depressive disorder. Psychol Med. 2012;42(4):671-81. https://doi.org/10.1017/S0033291711 001668.

24. Webb CA, Weber M, Mundy EA, Killgore WDS. Reduced gray matter volume in the anterior cingulate, orbitofrontal cortex and thalamus as a function of mild depressive symptoms: a voxel-based morphometric analysis. Psychol Med. 2014;44(13):2833-43. https://doi.org/10.1017/S0033291714000348.

25. Li J, Wang Z, Hwang JW, Zhao B, Yang X, Xin S, et al. Anatomical brain difference of subthreshold depression in young and middle-aged individuals. Neurolmage Clin. 2017;14:546-51. https://doi.org/10.1016/.jnicl.2017.02.022.

26. Takamiya A, Kishimoto T. Liang $\mathrm{K}$ ching, et al. thalamic volume, resting-state activity, and their association with the efficacy of electroconvulsive therapy. J Psychiatr Res. 2019;117:135-41. https://doi.org/10.1016/j.jpsychires.2019.08. 001.
27. Buckner RL, DiNicola LM. The brain's default network: updated anatomy, physiology and evolving insights. Nat Rev Neurosci. 2019;20(10):593-608. https://doi.org/10.1038/s41583-019-0212-7.

28. Pinault D. The Thalamic Reticular Nucleus: Structure, Function and Concept. Brain Res Brain Res Rev. 2004;46(1):1-31. https://doi.org/10.1016/j.bra inresrev.2004.04.008.

29. Haxby JV. Multivariate pattern analysis of fMRI: the early beginnings. Neuroimage. 2012;62(2):852-5. https://doi.org/10.1016/j.neuroimage.2012.03. 016.

30. Foland-Ross LC, Sacchet MD, Prasad G, Gilbert B, Thompson PM, Gotlib IH. Cortical thickness predicts the first onset of major depression in adolescence. Int J Dev Neurosci. 2015;46(1):125-31. https://doi.org/10.1016/j. ijdevneu.2015.07.007.

31. Liu F, Guo W, Yu D, et al. Classification of different therapeutic responses of major depressive disorder with multivariatepattern analysis method based on structural MR scans. PLoS One. 2012;7(7). https://doi.org/10.1371/journal.pone.0040968.

32. Zeng $L L$, Shen $H$, Liu L, Wang $L$, Li B, Fang $P$, et al. Identifying major depression using whole-brain functional connectivity: a multivariate pattern analysis. Brain. 2012;135(5):1498-507. https://doi.org/10.1093/brain/aws059.

33. Cao L, Guo S, Xue Z, Hu Y, Liu H, Mwansisya TE, et al. Aberrant functional connectivity for diagnosis of major depressive disorder: a discriminant analysis. Psychiatry Clin Neurosci. 2014;68(2):110-9. https://doi.org/10.1111/pcn.12106.

34. Gao S, Calhoun VD, Sui J. Machine learning in major depression: from classification to treatment outcome prediction. CNS Neurosci Ther. 2018; 24(11):1037-52. https://doi.org/10.1111/cns.13048.

35. Sankar A, Zhang T, Gaonkar B, Doshi J, Erus G, Costafreda SG, et al. Diagnostic potential of structural neuroimaging for depression from a multiethnic community sample. BJPsych Open. 2016;2(04):247-54. https://doi. org/10.1192/bjpo.bp.115.002493.

36. MacMaster FP, Carrey N, Langevin LM, Jaworska N, Crawford S. Disorderspecific volumetric brain difference in adolescent major depressive disorder and bipolar depression. Brain Imaging Behav. 2014;8(1):119-27. https://doi. org/10.1007/s11682-013-9264-X.

37. Redlich R, Opel N, Grotegerd D, Dohm K, Zaremba D, Bürger C, et al. Prediction of individual response to electroconvulsive therapy via machine learning on structural magnetic resonance imaging data. JAMA Psychiatry. 2016;73(6):557-64. https://doi.org/10.1001/jamapsychiatry.2016.0316.

38. Costafreda SG, Khanna A, Mourao-Miranda J, Fu CHY. Neural correlates of sad faces predict clinical remission to cognitive behavioural therapy in depression. Neuroreport. 2009;20(7):637-41. https://doi.org/10.1097/WNR. ob013e3283294159.

39. Bürger C, Redlich R, Grotegerd D, Meinert S, Dohm K, Schneider I, et al. Differential abnormal pattern of anterior cingulate gyrus activation in unipolar and bipolar depression: an fMRI and pattern classification approach. Neuropsychopharmacology. 2017;42(7):1399-408. https://doi. org/10.1038/npp.2017.36

40. Frangou S, Dima D, Jogia J. Towards person-centered neuroimaging markers for resilience and vulnerability in bipolar disorder. Neuroimage. 2017;145(Pt B):230-7. https://doi.org/10.1016/j.neuroimage.2016.08.066.

41. Rive MM, Redlich R, Schmaal L, Marquand AF, Dannlowski U, Grotegerd D, et al. Distinguishing medication-free subjects with unipolar disorder from subjects with bipolar disorder: state matters. Bipolar Disord. 2016;18(7):61223. https://doi.org/10.1111/bdi.12446.

42. Redlich R, Almeida JR, Grotegerd D, et al. Brain morphometric biomarkers distinguishing unipolar and bipolar depression: a voxel-based morphometry-pattern classification approach. JAMA Psychiatry. 2014;71(11): 1222-30. https://doi.org/10.1001/jamapsychiatry.2014.1100.

43. Grotegerd D, Stuhrmann A, Kugel H, Schmidt S, Redlich R, Zwanzger P, et al. Amygdala excitability to subliminally presented emotional faces distinguishes unipolar and bipolar depression: an fMRI and pattern classification study. Hum Brain Mapp. 2014;35(7):2995-3007. https://doi. org/10.1002/hbm.22380.

44. Grotegerd D, Suslow T, Bauer J, Ohrmann P, Arolt V, Stuhrmann A, et al. Discriminating unipolar and bipolar depression by means of fMRI and pattern classification: a pilot study. Eur Arch Psychiatry Clin Neurosci. 2013; 263(2):119-31. https://doi.org/10.1007/s00406-012-0329-4.

45. Schmaal L, Marquand AF, Rhebergen D, van Tol MJ, Ruhé HG, van der Wee NJA, et al. Predicting the naturalistic course of major depressive disorder using clinical and multimodal neuroimaging information: a multivariate pattern recognition study. Biol Psychiatry. 2015;78(4):278-86. https://doi. org/10.1016/j.biopsych.2014.11.018. 
46. Marquand A, Howard M, Brammer M, Chu C, Coen S, Mourão-Miranda J. Quantitative prediction of subjective pain intensity from whole-brain fMRI data using Gaussian processes. Neuroimage. 2010;49(3):2178-89. https://doi. org/10.1016/j.neuroimage.2009.10.072

47. Schrouff J, Monteiro JM, Portugal L, Rosa MJ, Phillips C, Mourão-Miranda J. Embedding anatomical or functional knowledge in whole-brain multiple kernel learning models. Neuroinformatics. 2018;16(1):117-43. https://doi. org/10.1007/s12021-017-9347-8.

48. Johnson-Lawrence V, Scott JB, James SA. Education, perceived discrimination and risk for depression in a southern black cohort. Aging Ment Health 2019;0(0):1-7. doi:https://doi.org/10.1080/13607863.2019.164 7131, 24, 11.

49. Pearson RM, Evans J, Kounali D, Lewis G, Heron J, Ramchandani PG, et al. Maternal depression during pregnancy and the postnatal period risks and possible mechanisms for offspring depression at age 18 years. JAMA Psychiatry. 2013;70(12):1312-9. https://doi.org/10.1001/jamapsychiatry.2 013.2163.

50. Chang-Quan H, Zheng-Rong W, Yong-Hong L, Yi-Zhou X, Qing-Xiu L. Education and risk for late life depression: a meta-analysis of published literature. Int J Psychiatry Med. 2010;40(1):109-24. https://doi.org/10.2190/ PM.40.1.i.

51. Ladin K. Risk of late-life depression across 10 European Union countries: deconstructing the education effect. J Aging Health. 2008;20(6):653-70. https://doi.org/10.1177/0898264308321002.

52. Park AL, Fuhrer R, Quesnel-Vallée A. Parents' education and the risk of major depression in early adulthood. Soc Psychiatry Psychiatr Epidemiol. 2013; 48(11):1829-39. https://doi.org/10.1007/s00127-013-0697-8.

53. Yan CG, Di Wang X, Zuo XN, Zang YF. DPABl: Data Processing \& Analysis for (resting-state) brain imaging. Neuroinformatics. 2016;14(3):339-51. https:// doi.org/10.1007/s12021-016-9299-4.

54. Ma X, Liu J, Liu T, Ma L, Wang W, Shi S, et al. Altered resting-state functional activity in medication-naive patients with first-episode major depression disorder vs. healthy control: a quantitative meta-analysis. Front Behav Neurosci. 2019;13(May):1-11. https://doi.org/10.3389/fnbeh.2019.00089.

55. Hao HH, Chen C, Mao WB, Zhong JG, Dai ZY. Aberrant brain regional homogeneity in first-episode drug-naïve patients with major depressive disorder: a voxel-wise meta-analysis. J Affect Disord. 2019;245:63-71. https:// doi.org/10.1016/j.jad.2018.10.113.

56. Song Y, Shen X, Mu X, Mao N, Wang B. A study on BOLD fMRI of the brain basic activities of MDD and the first-degree relatives. Int J Psychiatry Clin Pract. 2020;0(0):1-9. https://doi.org/10.1080/13651501.2020.1744663.

57. Yang $Y$, Liu $S$, Jiang $X$, et al. Common and specific functional activity features in schizophrenia, major depressive disorder, and bipolar disorder. Front Psychiatry. 2019;10(FEB):1-10. https://doi.org/10.3389/fpsyt.2019.00052.

58. Gray JP, Müller VI, Eickhoff SB, Fox PT. Multimodal abnormalities of brain structure and function in major depressive disorder: a meta-analysis of neuroimaging studies. Am J Psychiatry. 2020;177(5):422-34. https://doi.org/1 0.1176/appi.ajp.2019.19050560.

59. Li G, Rossbach K, Zhang A, Liu P, Zhang K. Resting-state functional changes in the precuneus within first-episode drug-naive patients with MDD. Neuropsychiatr Dis Treat. 2018;14:1991-8. https://doi.org/10.2147/NDT.S1 68060.

60. Zou QH, Zhu CZ, Yang Y, Zuo XN, Long XY, Cao QJ, et al. An improved approach to detection of amplitude of low-frequency fluctuation (ALFF) for resting-state fMRI: fractional ALFF. J Neurosci Methods. 2008;172(1):137-41. https://doi.org/10.1016/j.jneumeth.2008.04.012.

61. Lui S, Huang X, Chen L, Tang H, Zhang T, Li X, et al. High-field MRI reveals an acute impact on brain function in survivors of the magnitude 8.0 earthquake in China. Proc Natl Acad Sci. 2009;106(36):15412-7. https://doi. org/10.1073/pnas.0812751106.

62. Biswal B, Zerrin Yetkin F, Haughton VM, Hyde JS. Functional connectivity in the motor cortex of resting human brain using echoplanar mri. Magn Reson Med. 1995;34(4):537-41. https://doi.org/10.1002/ mrm.1910340409.

63. Liu F, Guo W, Liu L, Long Z, Ma C, Xue Z, et al. Abnormal amplitude lowfrequency oscillations in medication-naive, first-episode patients with major depressive disorder: a resting-state fMRI study. J Affect Disord. 2013;146(3): 401-6. https://doi.org/10.1016/j.jad.2012.10.001.

64. Schrouff J, Rosa MJ, Rondina JM, Marquand AF, Chu C, Ashburner J, et al. PRoNTo: pattern recognition for neuroimaging toolbox. Neuroinformatics. 2013;11(3):319-37. https://doi.org/10.1007/s12021-013-9178-1.
65. Hewing L, Kabzan J, Zeilinger MN. Cautious Model Predictive Control Using Gaussian Process Regression. IEEE Trans Control Syst Technol. 2019;PP:1-8. https://doi.org/10.1109/TCST.2019.2949757.

66. Fan L, Li H, Zhuo J, Zhang Y, Wang J, Chen L, et al. The human Brainnetome atlas: a new brain atlas based on connectional architecture. Cereb Cortex. 2016;26(8):3508-26. https://doi.org/10.1093/cercor/bhw157.

67. Guillery RW, Sherman SM. Thalamic relay functions and their role in corticocortical communication: generalizations from the visual system. Neuron. 2002;33(2):163-75. https://doi.org/10.1016/S0896-6273(01)00582-7.

68. Shadrina M, Bondarenko EA, Slominsky PA. Genetics factors in major depression disease. Front Psychiatry. 2018;9:73-83.

69. Zang YF, Yong H, Chao-Zhe Z, et al. Altered baseline brain activity in children with ADHD revealed by resting-state functional MRI. Brain Dev. 2007;29(2):83-91. https://doi.org/10.1016/j.braindev.2006.07.002.

70. Shu Y, Kuang L, Huang Q, He L. Fractional amplitude of low-frequency fluctuation (fALFF) alterations in young depressed patients with suicide attempts after cognitive behavioral therapy and antidepressant medication cotherapy: a resting-state fMRI study. J Affect Disord. 2020;276:822-8. https://doi.org/10.1016/j.jad.2020.07.038.

71. Patel MJ, Khalaf A, Aizenstein HJ. Studying depression using imaging and machine learning methods. Neurolmage Clin. 2016;10:115-23. https://doi. org/10.1016/j.nicl.2015.11.003.

\section{Publisher's Note}

Springer Nature remains neutral with regard to jurisdictional claims in published maps and institutional affiliations.
Ready to submit your research? Choose BMC and benefit from:

- fast, convenient online submission

- thorough peer review by experienced researchers in your field

- rapid publication on acceptance

- support for research data, including large and complex data types

- gold Open Access which fosters wider collaboration and increased citations

- maximum visibility for your research: over $100 \mathrm{M}$ website views per year

At BMC, research is always in progress.

Learn more biomedcentral.com/submissions 